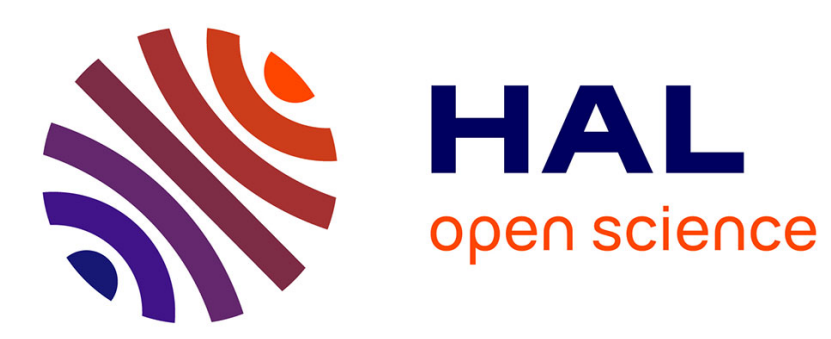

\title{
Les migrantes péruviennes au Chili et le travail du care: opportunités et risques d'une situation en plein essor
}

\author{
Juan Matas
}

\section{To cite this version:}

Juan Matas. Les migrantes péruviennes au Chili et le travail du care: opportunités et risques d'une situation en plein essor. Revue des Sciences sociales, 2014, Vers une société du CARE?, 52, pp.28-40. hal-01295019

\section{HAL Id: hal-01295019 \\ https://hal.science/hal-01295019}

Submitted on 30 Mar 2016

HAL is a multi-disciplinary open access archive for the deposit and dissemination of scientific research documents, whether they are published or not. The documents may come from teaching and research institutions in France or abroad, or from public or private research centers.
L'archive ouverte pluridisciplinaire HAL, est destinée au dépôt et à la diffusion de documents scientifiques de niveau recherche, publiés ou non, émanant des établissements d'enseignement et de recherche français ou étrangers, des laboratoires publics ou privés. 
REVUE DES

SGIENGES

SOMMAIRE

VERS UNE SOCIÉTÉ DU CARE? 2014 n52

PRÉSENTATION

p.4 BERNARD WOEHL, PHILPPE HAMMAN \& FREDDY RAPHAËL

p. 8 JUAN MATAS

p.80 CAROLINE GIACOMONI, HÉLEENE HOARAU

.12 DANIEL BERTAUX \& CATHERINE DEL CROIX

DES FLUX MIGRATOIRES DE TRAVAILLEUSES DU CARE

p. 18 HELMA WTZ \& EWA PALENGA-MÓLLENBECK divisée. Liens et transnationa

JUAN MATAS

(n)

D. 42 NATACHA BORGEAUD-GARCIANDA Relations de care, affects ef dominations. Le care à

FRANCE -ALLEMAGNE

ÉTUDES COMPARATIVES DE FORMES DE CARE

D.54 JANINA GLAESER

Assistmilesen methe et politiques de la petite entance : une comparaiso France-Allemagne

p.62 JULLE SENTIS

Apprendre les métiers du care en France et en Allemagne

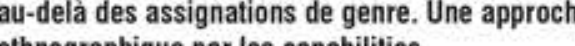

LE CARE EN INSTITUTION

TROIS ÉTUDES DE CAS EN FRANCE

p.72 MOUNRFARHAT

MOUNIR FARHAT personnel et distance professionnelle en unité Alzheimer

P. 80 CAROLINE GIACOMONI, HÉLEENE HOARAU
\& ALAIN MONNEREAU Enjeux et conflits autour du care à travers l'expérience de la délégation à domicile des traitents en concérologie

p. 86 LAURELINE COULOMB Léthique du care malmenée au cours des interactions entre infirmiers et individus sans-abri LUTTES ET RÉFORMES POTENTIELLES

p. 94 URSULA APITZSCH net l'ordre de genre

p. 102 AGNËS BERTHELOT-RAFFARD La discrimination par association : une expression du care dominé

p. 110 CHANTAL NICOLE-DRANCOUR Pour une reconnaissance du care dans des sociétés de pleine activité

p. 118 DANEL BERTAUXX

政 CHANTIERS DE RECHERCHE

p. 132 ÉLOOIE VALENTIN

Emotions, dynamiques citoyennes et espace public. L'expérience du projet social d'une maison de quartier a Dunkerque

p. 142 SUZY GUTH Conflit dans le vignoble : Ammerschwihr et l'AOC Kaefferkopf

LU - À LIRE

p. 154 Recensions

p. 166 Résumés des articles

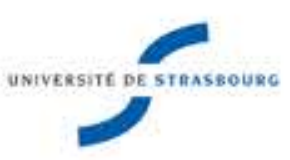

Cirs

Revve bénéficiant du soutien de l'Isstitut
des Sciences Humaines el Sociales du CNRS
SSN 1623-6572

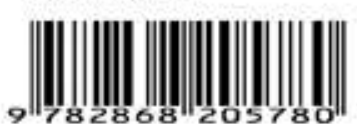

REVUE DES SOIENOES SOBALES

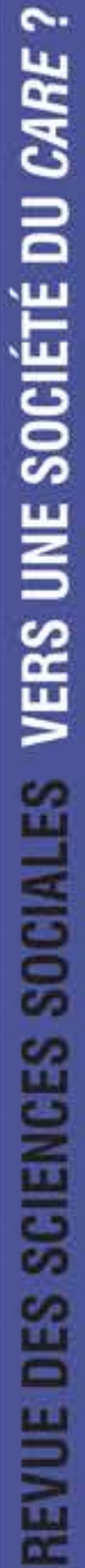

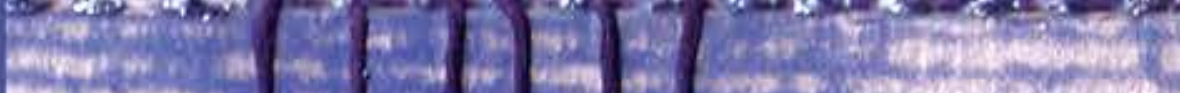

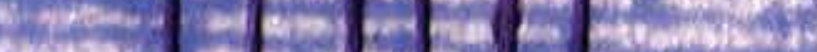

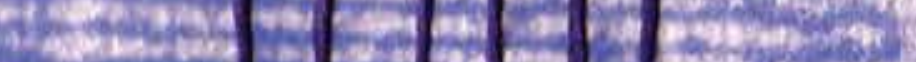
6.

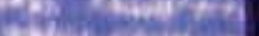
wast:

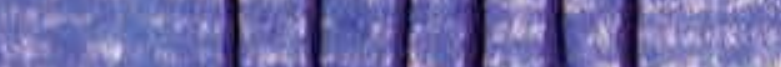

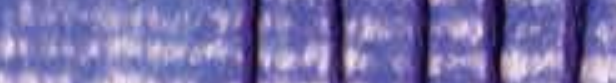

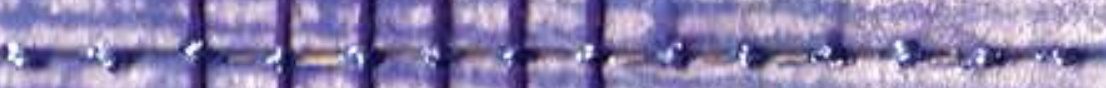
-

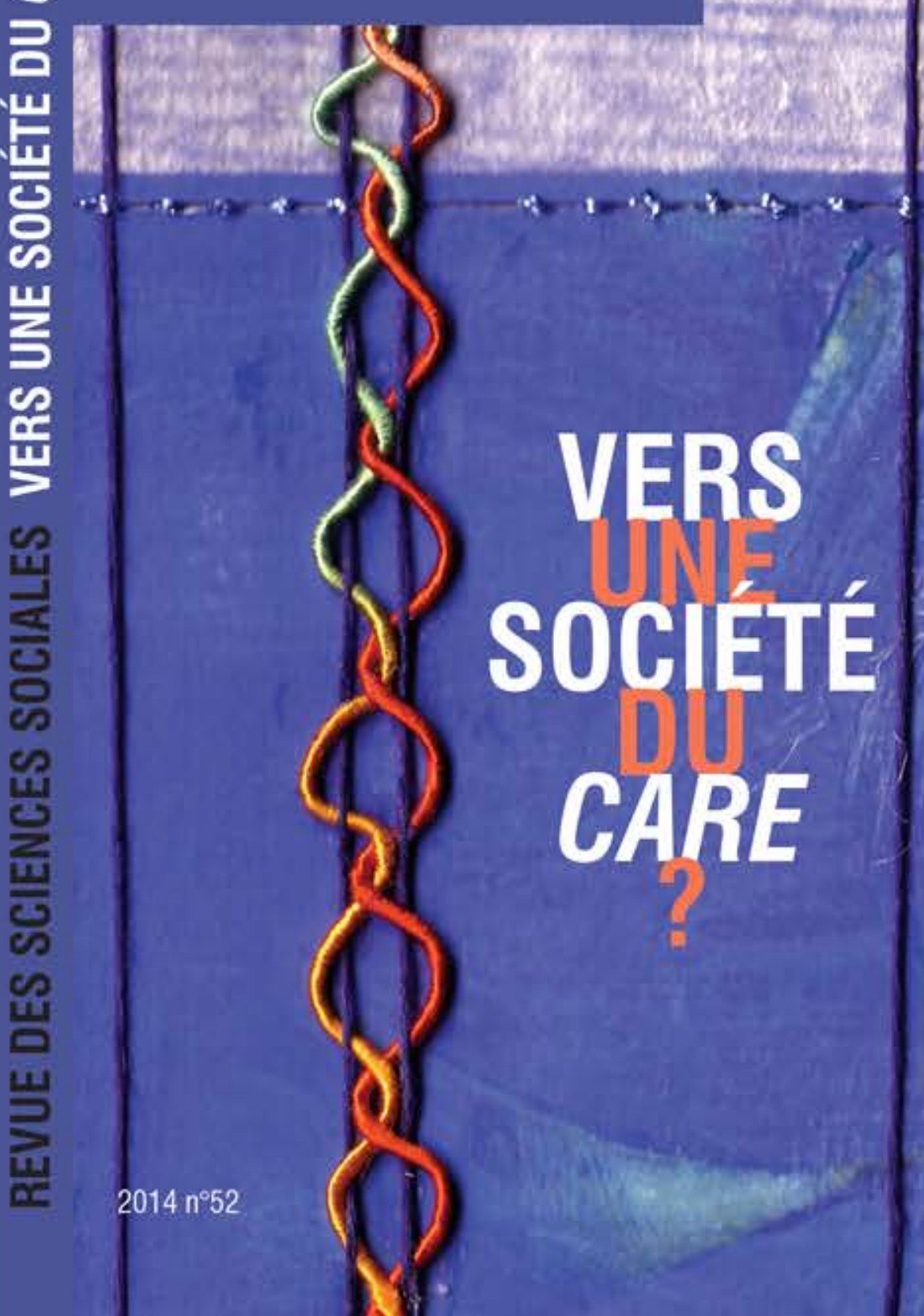


REVUE DES

SAIN

SOGALES

VERS UNE SOCIÉTÉ DU CARE ?

$2014 n^{\circ 5}$ ?

- Directeur scientifique

Freddy Raphaël

- Rédacteur en che

Patrick Ténoudji

- Comité scientifique

Gerges Balandier (EHESS Paris)

(CENRS Toulouse).

(MaymTP Paris) Gionas Paris), Jean Cuisenier

Milano). Jose Carios Gomes da Silva (Portugal).

François Hèran (INED Paris), Claude Javeau (Univ. Libre

de Bruxelles). Nicole Lapierre (EHESS/CNRS Paris).

Marianne Mesnil (Univ. Libre de Bruxelles).

Sonia Montecino (Univ. de Chile), Jean Rèmy (Univ.

Alain Tarrius (Univ. Toulouse-Le Mirail), Alain Touraine

(CEMS Paris)

- Comité de rédaction

Chelle Biannuis-Gasser (Univ. Tours), Maurice Blane Univ. Strasbourg), Nicoletta Diasio (Univ. Strasbourg). Woltong Strasbourg). Antida Gazzola (Univ. Genova). Philippe Hamman (Univ. Strasbourg). Pascal Hintermeyer (Univ. Strasbourg), Leila Jeolas (Uniy Londrina), Reinhard Johler (Univ, Túbingen). Salvatore La Mendola (Univ. Padova), David Le Breton (Univ. Strasbourg/UF), Juan Malas (Univ. Strasbourg. Gabriele Profita (Univ. Palerme), Ilario Rossi (Univ. Lausanne), Patrick Schmoll (CNRS Strasbourg), Roger Some (Uni. Slasbourg), Simona tersign

Collaborateurs éditoriaux

Anny Bloch, Marie-Noële Dents,

Anny Bloch, Marie--Noële Der
- Équipe iconographique sur ce numéro

Vincent Hans, Aline Mathy, Patrick Schmo

- Ce numero a été coordonné par :

Catherine Delcroix, Juan Matas \& Daniel Bertaux

- Maquette

Couverture : Aline Mathy

Presses Universitimires de Stroshour

- Administration

Presses Universitaires de Strasboutro

5 allée du Gal Rouvillois - CS 50008

FR-67083 Strasbourg Cedex

Tél. 0368856265

e-mail : pu-strasbourg@unistra.tr

- Diftusion/distribution

agences d'abonnement :

cid@msh-paris.tr

(nstitutions:

www.lcdpu.fi

18 rue Robert-Schuman

CS 90003

FR-94227 Charenton-le-Pont Ceder

Tel. 0153485630

Revue publiée par la Faculté des sciences sociale

de TUniversité de Strasbourg et le laboratioire

DST 1023.0572 (2)

www.revue-des-sciences-sociales.com
Ál'attention des auteurs

Les articles sont à adresser par document attachẻ à l'attention de Patrick Ténouditi, rédacteur en chêt <patrick.tenoudji@misha.fr>

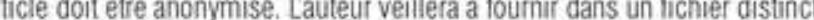

- sestitres ou protession et son appantenance instiutionnelle (université, centre de recherche)

- un résumé d'une dizaine de lignes en français et en anglais.

Le texte comporte au maximum 40000 signes et blancs, notes et bibliographie comprises. II est Myhme par des intertitres courts, avec deux niveaux dintertitres au maximum. Les passages cites de rextes en langues tlangertes sont traduns en lançais. Les nolts, oni

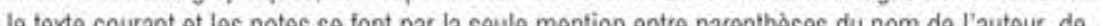
l'année de publication et, le cas échéant, des numéros de pages citées.

Exemples

- ... a ainsi que lindique J. Dupont (2003a) ...

-.... cette question a lairt objel de plusieurs travaux (Oupont 2003a, Durand 2004. 2007) ...

La bibliographie suit les consignes de présentations suivantes :

- pour un ouvage . Oupont J. (2003a), Thre de rouvrage en iltafique, Lieu d'édition, Editeur.

- pour un chapitse douvrage : Durand M. (2004). Titre du chapitre sans guillemets, in Dupont

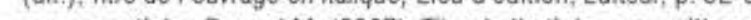

-

Les articles soumis par les auteurs sont examinés chacun par un comité d'au moins deux lecteurs qui rendent des avis séparés. Lexemplaire du texte qui leur est remis ne mentionne pas lidentité de Tauteur, et lauteur de son cote ne peut se laire communiquer T identitie de ses lecteurs : la decision

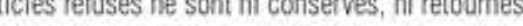

Si des graphiques et illustrations sont nécessaires à l'appui du texte, l'auteur en adresse les originaux ou les fichiers image haute résolution (300dpi) à la rédaction. Hors ce cas de figure, la recherche la rédaction de la revue. 


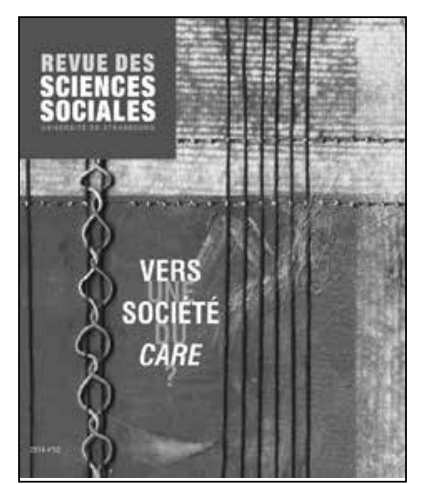

Khadija Seddiki

artiste plasticienne, diplômée de l'Académie royale des beaux-arts de Belgique, de l'Académie des arts et métiers

Constantin Meunier d'Etterbeek, des Écoles supérieures des beaux-arts d'Alger et d'Oran, et de l'Université Denis Diderot-Paris VII. Elle a fondé TISSART, école d'art et atelier de tissage, à Vanves. http://khadijaseddiki.com/
Dans les sociétés du "Nord" de la planète, les besoins de care augmentent alors que les femmes n'acceptent plus guère de s'y consacrer sans contrepartie. Ce déséquilibre engendre des flux importants et différenciés de migrantes venant de «l'Est» ou du «Sud». Qui sont-elles, dans quelles conditions viennent-elles, dans quels cadres, lieux, rapports et conditions concrètes travaillent-elles, quels sont leurs droits?

Ce numéro examine la situation de travailleuses du care: Polonaises en Allemagne, Péruviennes à Buenos Aires ou au Chili; il aborde les contextes institutionnels de ces activités en France, en Allemagne, dans une perspective comparative. Une "société du care» est-elle en train d'émerger?
Couverture

image: Khadija Seddiki composition: Aline Mathy 


\section{PRÉSENTATION}

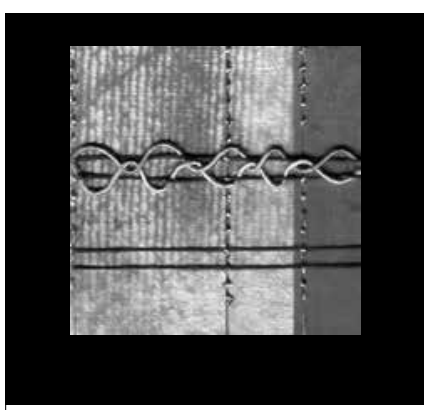

4

BERNARD WOEHL

PHILIPPE HAMMAN \& FREDDY RAPHAËL

Hommage

à François Steudler 8

JUAN MATAS

Introduction

12

DANIEL BERTAUX \& CATHERINE DELCROIX

Présentation
DES FLUX MIGRATOIRES DE TRAVAILLEUSES DU CARE

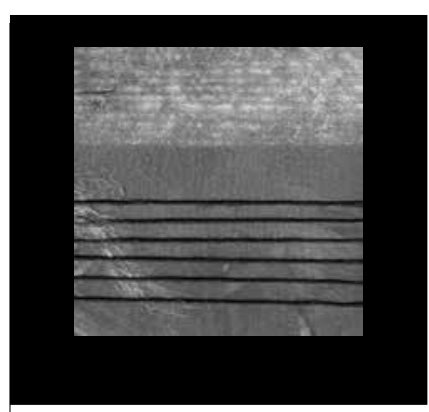

18

HELMA LUTZ \&

EWA PALENGA-

MÖLLENBECK

Les migrantes du care dans

l'Europe divisée. Liens et

contradictions dans un

espace transnational

\section{8}

\section{JUAN MATAS}

Les migrantes péruviennes au Chili et le travail de care

opportunités et risques

d'une situation en plein essor

\section{2}

NATACHA BORGEAUD-

\section{GARCIANDA}

Relations de care, affects et dominations. Le care à demeure à Buenos Aires
FRANGE -ALLEMAGNE : ÉTUDES COMPARATIVES DE FORMES DE CARE

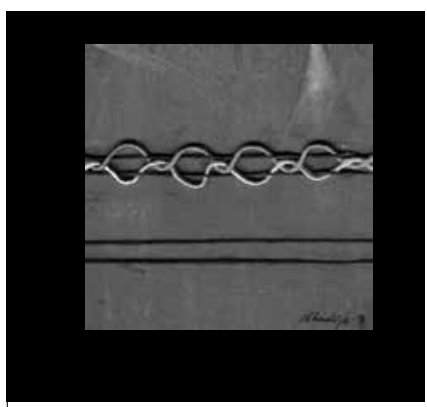

54

\section{JANINA GLAESER}

Assistant(e)s maternel(le)s d'origine étrangère et politiques de la petite enfance: une comparaison France-Allemagne

\section{2}

\section{JULIE SENTIS}

Apprendre les métiers du care en France et en Allemagne au-delà des assignations de genre. Une approche ethnographique par les capabilities
LE GARE EN INSTITUTION : TROIS ÉTUDES DE CAS EN FRANGE

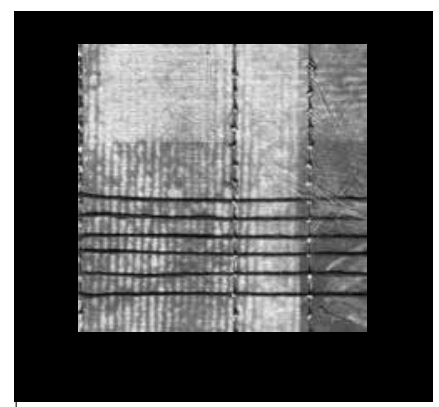

72

\section{MOUNIR FARHAT}

Un dilemme du care:

tension entre engagement personnel et distance professionnelle en unité Alzheimer

80

CAROLINE GIACOMONI, HÉLĖNE HOARAU \& ALAIN MONNEREAU

Enjeux et conflits

autour du care à travers

l'expérience de la délégation à domicile des traitements en cancérologie

86

\section{LAURELINE COULOMB}

L'éthique du care malmenée au cours des interactions entre infirmiers et individus sans-abri 


\section{LUTTES ET RÉFORMES POTENTIELLES}

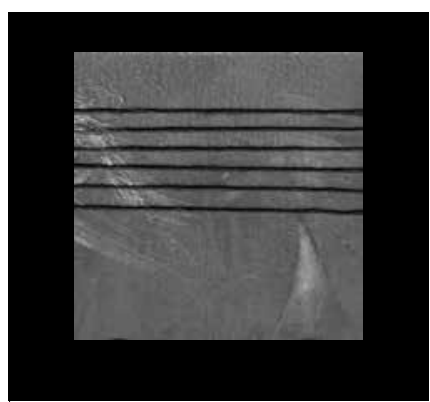

94

URSULA APITZSCH

Le care, la migration

et l'ordre de genre

102

AGNÈS BERTHELOT-RAFFARD

La discrimination

par association:

une expression

du care dominé

110

CHANTAL NICOLE-

\section{DRANCOURT}

Pour une reconnaissance

du care dans des sociétés

de pleine activité

\section{8}

\section{DANIEL BERTAUX}

Le care comme partie

émergée de la production

de la vie

\section{CHANTIERS} DE RECHERCHE

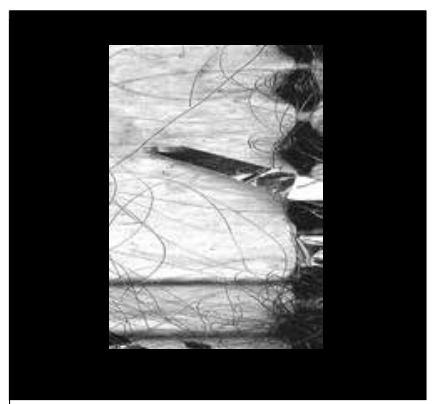

132

ELODIE VALENTIN

Émotions, dynamiques

citoyennes et espace public. L'expérience du projet social

d'une maison de quartier à

Dunkerque

\section{2}

\section{SUZY GUTH}

Conflit dans le vignoble:

Ammerschwihr

et I'AOC Kaefferkopf
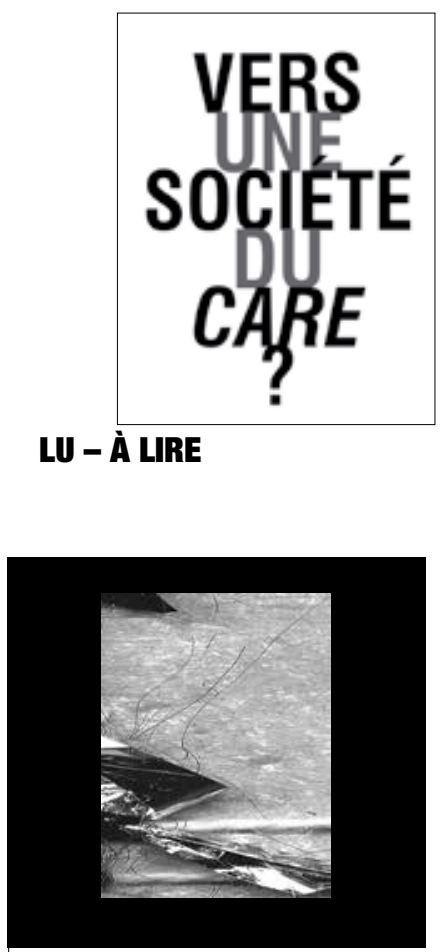

154

RECENSIONS

166

RÉSUMÉS DES ARTICLES 


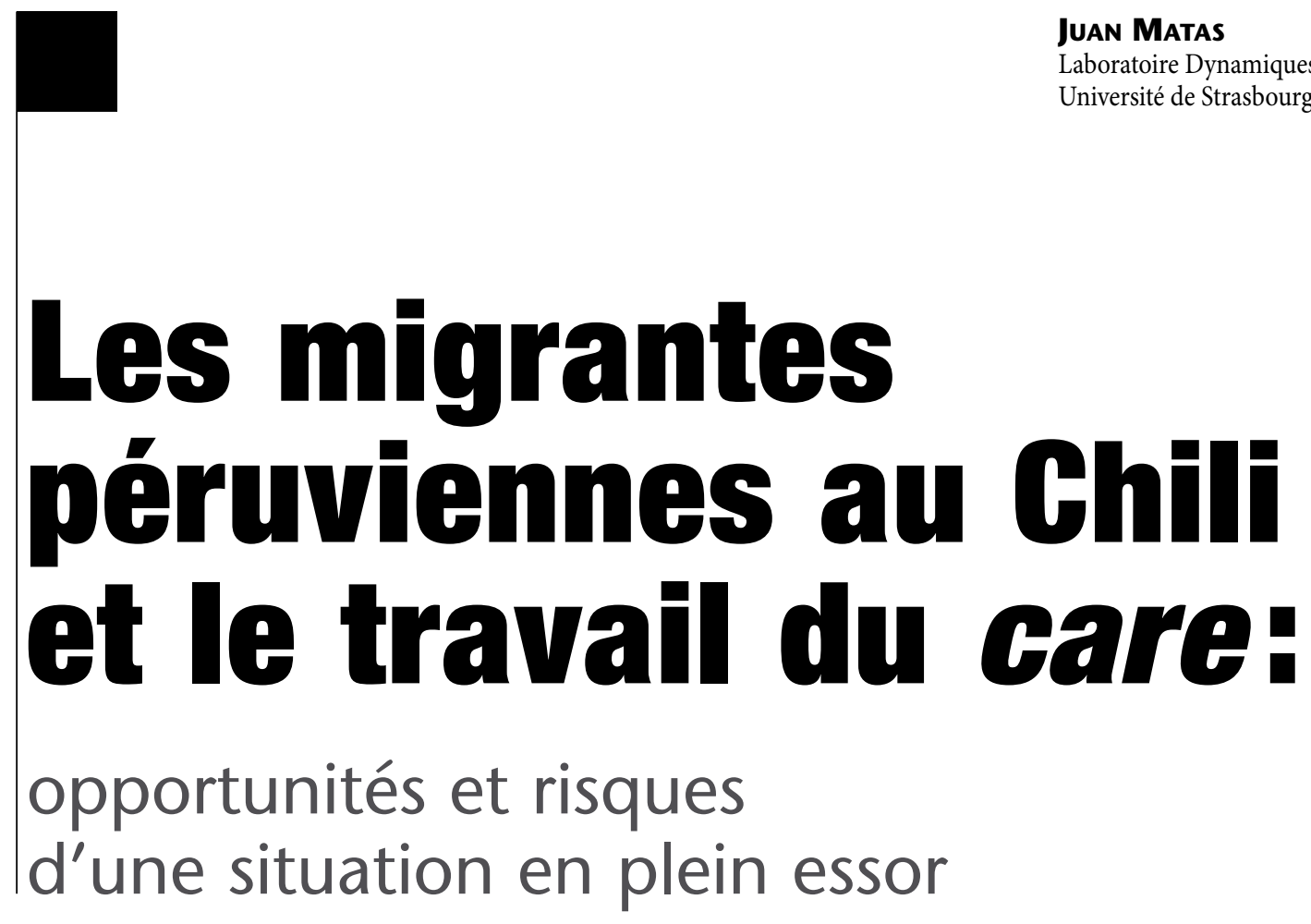

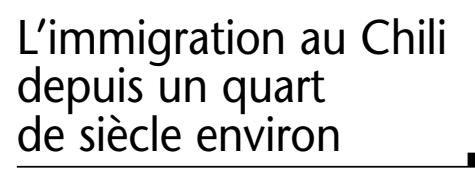

S'intéresser aux mouvements migratoires fournit un excellent mode d'entrée pour comprendre les évolutions qui ont lieu dans une société donnée à un moment donné de son histoire. En général, les temps de prospérité et de forte cohésion sociale correspondent avec une émigration faible et peuvent donner lieu à une immigration plus ou moins importante, alors que ceux de crise, de difficultés et de désordres donnent plutôt lieu au phénomène inverse. Les formes que prennent les migrations, les pays d'où viennent les immigrants ou ceux vers lesquels se dirigent les émigrants, la durée du cycle migratoire ou le profil des populations concernées peuvent se modifier au fil du temps mais un pays est plutôt d'émigration quand il se range plutôt dans la catégorie des "pays pauvres» ou qu'il est frappé par des troubles graves, et il est plutôt d'immigration lorsqu'il fait partie des "pays riches» et que sa situation est considérée comme satisfaisante du point de vue de la sécurité ressentie par ses habitants. Bien sûr, ceci n'est pas suffisant pour déclencher l'immigration ou l'émigration, mais cela semble être un facteur majeur dans ce domaine. Parmi les autres éléments importants, je retiendrai l'évolution des indicateurs sociodémographiques, qui rendent compte des besoins objectifs, tant pour des individus ou des groupes que pour la société tout entière: là où se fait sentir une pénurie de main-d'œuvre il y a souvent une société avec une faible natalité, un allongement rapide de l'espérance de vie et un vieillissement global de la population - ce qui est la situation ordinaire des sociétés les plus aisées -, avec comme corollaire le besoin d'attirer une population allochtone, alors que là où le chômage et le sous-emploi concernent une partie importante de la population du pays (et notamment des jeunes) on a souvent affaire à des pays qui ne sont pas encore sortis de la transition démographique, où les inégalités sont souvent fortes et où l'émigration fait office de soupape pour éviter des explosions dues à la pauvreté et au mécontentement d'une partie significative de la population.

On ne peut pas s'attarder ici sur l'histoire migratoire du Chili, mais il est nécessaire d'en dire quelques mots pour resituer notre propos global. Si le premier peuplement du continent remonte à l'arrivée des premiers peuples asiatiques qui franchirent le détroit de Behring (il y a de cela entre 40000 et 100000 ans, selon les sources), et à 11000 ans environ pour le Chili, le grand tournant de l'histoire chilienne se situe au moment de l'expédition de Diego de Almagro, en 1536, puis de la conquête entreprise par celui-ci, poursuivie par Pedro de Valdivia, qui fonde la ville de Santiago de la Nouvelle Extrémadoure en 1542, ouvrant ainsi la voie à la colonisation (voir, à ce sujet, les ouvrages de Nicolás Sánchez-Albornoz, La población de América latina. Desde los tiempos precolombinos al año 2000, (1977) Madrid, Alianza éd., 2e édition, de José Bengoa, La emergencia indígena 
en América latina, (2000) Santiago, Fondo de Cultura Económica éd., et de Sergio Villalobos, Chile y su historia, (2008) Santiago, Editorial Universitaria, $11^{\mathrm{e}}$ édition). C'est en 1810 que fut proclamée l'indépendance du Chili et en 1818 qu'elle devint une réalité. $\mathrm{Du}$ point de vue de l'immigration, ceci signifie qu'elle ne sera plus contrôlée par les autorités espagnoles et on verra ainsi l'élargissement des zones géographiques d'où sont issus les immigrés, mais il faudra attendre les années $1860-1870$ pour voir un essor de cette immigration, bien plus modeste qu'en Argentine, au Brésil ou en Uruguay mais bien réel au demeurant. De ces années-là jusque vers le début des années 1950, le Chili est resté largement excédentaire dans le rapport immigration / émigration, même si cette dernière n'a pas été marginale; de nombreux Chiliens ont émigré vers d'autres pays latinoaméricains, et surtout vers l'Argentine, mais aussi vers l'Amérique du Nord (les mineurs et autres "pirquineros» attirés par la ruée vers l'or en Californie ont donné lieu à de nombreux récits, tel celui de Joaquín Murieta, sorte de bandit romantique) ou, en quantité moindre, vers l'Australie. Ceci a contribué à créer le personnage du Chilien «pat'e perro ", surtout dans la mesure où le pays s'illustrait par l'existence d'une marine marchande reliant Valparaíso aux ports du Pacifique mais aussi d'Europe. Pour revenir à l'immigration, lors de cette période elle fut européenne pour l'essentiel (espagnole et italienne, surtout, mais aussi française, allemande, britannique, ou provenant d'Europe centrale et orientale: des Juifs et des Slaves, notamment), mais nombre de ces immigrés provenait également du Moyen-Orient (Syrie, Palestine, Liban) et même d'Extrême-Orient (Chine). Si la dimension économique était de loin la plus importante, il y a eu également une immigration produite par les persécutions politiques et religieuses (émigrés français chassés après la fin sanglante de la Commune, réfugiés espagnols après la guerre civile dans ce pays, Juifs d'Europe orientale victimes de pogroms et autres formes de ségrégation). L'immigration européenne répondait à une politique gouvernementale de peuplement du pays, où les arrière-pensées racistes n'étaient pas absentes («blanchir la population» ou «améliorer la race» étaient des expressions utilisées par les défenseurs de cette politique, qui avait aussi pour but de coloniser le pays Mapuche). Quant à l'immigration en provenance des autres pays latino-américains, elle était peu significative, si on excepte l'immigration étudiante (de nombreux étudiants de divers autres pays du sous-continent venant, jusqu'au début des années 1970, faire leurs études ou les compléter, plutôt à l'Université du Chili et, dans une moindre mesure, à l'Université Catholique à Santiago, et retournant après dans leurs pays respectifs pour la grande majorité d'entre eux) et de quelques exilés politiques de pays proches.

Avant de conclure sur ce point, il semble intéressant d'évoquer les nouvelles formes de métissage que cette immigration a provoqué (sur la question du métissage, voir l'ouvrage de Magnus Mörner, Le métissage dans l'histoire de l'Amérique latine (1971) Paris, Fayard éd., celui d'Alejandro Lipschutz, El problema racial en la conquista de América y el mestizaje, (1967) Santiago, Editorial Andrés Bello, et celui de Sonia Montecino, Madres y Huachos. Alegorías del mestizaje chileno, (1991) Santiago, Edit. Sudamericana). On ne peut pas oublier que le Chili est fondé sur le métissage indo-espagnol dont les débuts se situent au $\mathrm{XVI}^{\mathrm{e}}$ siècle; pendant toute la période coloniale, et même jusque vers 1860 , les autres apports furent très restreints. L'arrivée d'une population aux origines très diversifiées a modifié cette situation; au départ, les différentes colonies (terme utilisé pour désigner les diverses nationalités ou origines) ne se mélangèrent au reste de la population que de façon parcimonieuse, mais la seconde (et davantage encore la troisième) génération accomplit le parcours de l'intégration, de façon certes inégale. Les Allemands $\mathrm{du}$ Sud du pays, notamment, furent connus par leur forte endogamie matrimoniale et par le maintien de la langue et des traditions de leur pays d'origine, mais cette situation s'est, depuis, substantiellement atténuée. Dans l'ensemble, on peut dire que les différents groupes ont contribué à façonner une identité plus diversifiée, tout en intégrant cette chilenidad («chilianité », terme d'usage courant au Chili) qui donne à la nation une personnalité singulière dans la région (on pourrait en dire autant des processus de (re) construction d'une identité nationale de pays tels que le Brésil et l'Argentine, parmi d'autres). Il ne s'agit pas de nier les problèmes existants, et le premier de ceux-ci demeure le préjugé à l'égard de l'Indien, le Mapuche surtout. Il faut, cependant, reconnaître que l'intégration des groupes immigrés s'est faite de façon très poussée, même s'il faudrait en étudier les caractéristiques pour chacun des groupes. L'étude de l'immigration juive serait ici particulièrement riche d'enseignements, car celle-ci offre un exemple par le fait que sa spécificité religieuse, majoritairement maintenue, ne semble pas faire obstacle à son intégration dans la communauté nationale, comme en témoigne le nombre important de ses descendants qui occupe une place de premier plan dans les sciences, la politique ou la culture du Chili contemporain.

Telle était donc la situation du point de vue des mouvements migratoires jusqu'en 1973. Avec le coup d'État militaire, l'exil chilien va constituer une diaspora qui va de l'Australie à l'Europe, mais aussi les très nombreux Chiliens, ayant ou non le statut de réfugié, qui vont s'installer du Nord au Sud du continent américain, avec des groupes particulièrement importants au Brésil, au Venezuela, à Cuba, aux Etats-unis d'Amérique et au Canada, sans oublier le groupe de loin le plus nombreux, celui des Chiliens résidant en Argentine. Cette émigration est composée à la fois de ceux qui quittent le pays en tant que réfugiés et de ceux poussés à l'émigration par la difficile conjoncture économique interne, avec des situations également qui sont un mélange des deux causalités mentionnées. Précisons, toutefois, que la dictature de Pinochet ne fut pas la première à pousser à l'exil une population persécutée en raison de ses idées. Avant elle, 
la guerre civile de 1891, qui vit l'instauration de la République parlementaire, ou les années troublées qui virent la venue au pouvoir du général Carlos Ibañez en 1928, ou encore la mise hors la loi du Parti Communiste par le gouvernement de González Videla en 1948, ont conduit de nombreux Chiliens sur les chemins de l'exil, mais il ne fait pas de doute que l'ampleur de l'exil des années 1973-1989 n'a pas de commune mesure avec les antérieurs, comme les crimes, les violations des droits de l'Homme et la répression en général ont atteint un niveau sans précédent dans l'histoire du pays.

Depuis une bonne vingtaine d'années, une situation nouvelle est apparue. L'émigration chilienne a diminué, au fur et à mesure que le pays entrait à la fois dans une phase de normalisation politique et de développement économique. L'heure était plutôt, à partir de 1984-1985, au retour des exilés et de nombre d'émigrés économiques, mais aussi à l'arrivée d'un nouveau courant migratoire, composé majoritairement de ressortissants de pays voisins (surtout Péruviens) mais aussi de Coréens, les deux groupes revêtant, néanmoins, des caractéristiques bien différentes. Les premiers ont, pour une partie importante d'entre eux, un faible niveau d'études et de qualification et constituent une main-d'œuvre bon marché, plus présente (proportionnellement) dans les villes du Nord du pays mais que l'on retrouve également à Santiago; on peut noter que les femmes, parfois seules, sont de plus en plus nombreuses et qu'elles constituent désormais une fraction assez importante des personnels de maison (les bonnes péruviennes remplacent de plus en plus les jeunes femmes venues des campagnes du Sud du Chili pour s'embaucher comme domestiques). Ici, il y a un véritable clivage du point de vue du profil de ces femmes, de leur niveau d'études notamment, et donc du point de vue des aspirations et des trajectoires.

L'immigration a lieu, selon les pays et les époques, dans des contextes d'une extrême diversité, et elle revêt des significations qui diffèrent en fonction d'un grand nombre de paramètres. Néanmoins, ce phénomène est toujours une richesse par l'ouverture qu'il suppose pour les sociétés qui s'y trouvent confrontées, à condition de préserver la cohérence que toute société se doit de garder pour garantir à ses habitants, par-delà la diversité et même les aspects antagoniques que toute société comporte, le maintien d'une identité et d'un projet commun à tous ceux qui la conforment, façonnés à travers le temps, jamais immuables mais toujours présents. La participation des immigrés à la construction de l'édifice social, à la mémoire collective, est le plus sûr moyen de leur raccordement à la société d'accueil, avec les différences subsistant entre l'immigration temporaire et la définitive, qu'il faut prendre en compte même lorsque l'on sait les difficultés que suppose de les distinguer l'une de l'autre. Pour le Chili, né en tant que société de la rencontre de deux cultures, pour utiliser un langage qui véhicule des ambiguités mais qui ne manque pas de pertinence, l'immigration des $\mathrm{XIX}^{\mathrm{e}}$ et $\mathrm{XX}^{\mathrm{e}}$ siècles a enrichi son patrimoine culturel, a élargi ses horizons et a permis de sortir, un tant soit peu, du tête-à-tête colonisateur-colonisé. On peut penser que l'immigration d'aujourd'hui, qui reflète d'autres réalités, saura constituer un nouvel apport à l'édification de la chilianité du futur. C'est pourquoi il me semble important de voir comment cette question a été traitée par les gouvernements concertationnistes ${ }^{1}$ qu'a connu le Chili entre 1990 et 2010. Il faudra, sans doute, voir quels infléchissements résultent, en la matière, de l'arrivée au pouvoir de Sebastián Piñera, à la tête d'une coalition de droite, intervenue en mars 2010, et les transformations que ne manquera pas d'apporter l'élection récente de Michelle Bachelet, à la tête d'une «Concertation remaniée», baptisée Nueva Mayoría, pour la période 2014-2018, mais ce sera l'objet de travaux ultérieurs.

Pendant les vingt années allant de 1989 à 2009, quatre présidents ont été désignés par le suffrage universel, à la tête d'une coalition de centre-gauche (la Concertation pour la Démocratie), ayant de nombreux défis à relever pour une société chilienne qui devait effacer les traces d'une dictature sanglante et consolider la démocratie, redonner une cohésion à une société meurtrie, conjuguer croissance économique et équité sociale, consolider la régionalisation, développer une société civile moderne et participative ou encore choisir ses partenariats privilégiés à l'échelle internationale (association à Mercosur, relations avec ALENA, avec l'Union européenne, adhésion à APEC, etc.). Parmi les dossiers à ouvrir, celui de l'intégration des immigrés pouvait ne pas apparaître comme prioritaire, mais il est pour nous un bon analyseur, qui nous renseigne sur les orientations et la nature du pouvoir en place comme sur l'évolution des mentalités. À ce titre, il faut souhaiter que le débat sur la question migratoire, entamé au cours des dernières années, se poursuive et s'étende, car il sera nécessairement relié à celui de la transformation des mentalités dans bien d'autres domaines. Ayant eu l'occasion, en juillet/août 2001, de participer à Santiago au séminaire Exclusion sociale et politiques publiques, organisé par le CIEG (Centre Interdisciplinaire des Études de Genre, Université du Chili) et le Ministère Secrétariat Général de Gouvernement du Chili, j'ai vérifié la place importante que prend à l'heure actuelle la question de l'immigration (notamment péruvienne) dans certains segments de la société civile et même dans la réflexion conduite au niveau gouvernemental ou à celui, plus modeste, des travailleurs sociaux confrontés aux situations et aux revendications des immigrés et du monde associatif qui se mobilise pour les soutenir. La lecture d'une littérature croissante sur ce sujet et celle de la presse quotidienne, notamment durant l'année 2013, a pu me conforter dans l'idée de cette visibilisation, relativement nouvelle au Chili, de la «question immigrée».

Reprenons les termes utilisés par Eduardo Domenech, dans la présentation de l'article cité ici, concernant l'évolution des discours officiels sur les immigrés provenant des pays voisins et la signification de cette évolution en Argentine: «En se fondant sur des documents officiels et sur des interventions publiques de fonctionnaires gouvernementaux qui couvrent 
la période qui va de 2000 à 2008 , cet article suggère que, par opposition à la «rhétorique de l'exclusion» qui prévalait dans l'Argentine des années 1990, la "rhétorique de l'inclusion» qui prend forme à présent se distancie des représentations qui participent de la construction sociale de l'immigrant comme «menace», en mettant l'accent sur certains aspects de l'immigration qui renvoient aux notions d'apport, de contribution et d'avantage $»^{2}$. Passer d'une rhétorique à l'autre n'est pas sans effets sur les représentations collectives qu'a une société de l'immigré, et donc des comportements que cette société légitime à son égard. $D$ 'ailleurs, ce n'est probablement pas un hasard que les rhétoriques de l'exclusion aient été largement façonnées (ou renforcées) pendant les dictatures de part et d'autre de la Cordillère des Andes. Mais on peut noter qu'au Chili, sous l'administration Piñera qui a gouverné jusqu'en mars de cette année, le discours officiel est demeuré semblable à celui qui avait dominé sous les gouvernements de la Concertation: l'accent est plutôt mis sur les aspects positifs de l'immigration et sur l'importance de l'intégration, condamnant les actes xénophobes qui font, ici ou là, leur apparition (j'y reviendrai un peu plus loin).

Plusieurs raisons semblent indiquer que le phénomène de l'immigration croissante, composée surtout de ressortissants d'autres pays sudaméricains, n'est pas près de se tarir. D'abord, les tendances démographiques vont dans ce sens: la baisse de la fécondité (on est passés de 4,95 enfants par femme en 1950-1955 à 2,67 en 1980-1985 et à 2 en 20002005), ou encore le vieillissement de la population (on avait une espérance de vie à la naissance de 54,8 ans en 19501955, de 70,7 ans en 1980-1985 et de 77,7 ans en 2000-2005, avec une différence entre les hommes et les femmes qui est passée de moins de 4 ans à 6 ans pour la dernière période étudiée). On peut noter que la mortalité infantile est passée, lors de la même période, de $120,3 \%$ à $18,4 \%$, puis à $8 \%$, ce qui contribue à expliquer la modification des comportements en matière de natalité. Il y a aussi, pour alimenter cette migration, l'accroissement de la distance en matière de croissance économique et de développement avec la plupart des autres pays sud-américains, qui rend le Chili plus attractif comme destination pour de nombreux candidats à l'émigration (notamment au Pérou ou en Bolivie). En somme, les facteurs qui sont à l'origine de l'offre et de la demande en matière d'immigration sont là. (Les chiffres que je donne ici sont tirés du document Chile: proyecciones y estimaciones de población. Total País. 1950-2050, publié par INE et CEPAL en 2009).

Cependant, cette immigration, dont les origines géographiques tendent à se diversifier, avec une présence accrue de nouvelles nationalités, ne va pas sans poser des problèmes, aussi bien pour la société chilienne que pour les immigrés eux-mêmes. Nous reviendrons sur cette question un peu plus loin. Voyons maintenant quelques éléments des politiques mises en place pour l'accueil et l'intégration des populations étrangères.

Selon les données actualisées en décembre de 2008 par le Departamento de Extranjería y Migración (DEM) du Ministère de l'Intérieur chilien, le nombre d'immigrants atteignait 317057 personnes, ce qui équivaut à $1,8 \%$ du total de la population du pays $^{3}$.

Le même document résume de façon claire et concise un certain nombre d'orientations des quatre mandatures concertationnistes (bien que pour la dernière il se contente d'évoquer ce que devraient en être les grandes lignes, car il a été rédigé au début du premier mandat de la présidente Bachelet). Dans un premier temps, le document rappelle les grandes tendances du phénomène migratoire: «Les chiffres de migration au Chili au $\mathrm{xx}^{\mathrm{e}}$ siècle permettent de conclure que ce phénomène a connu des changements qui peuvent être perçus d'un simple coup d'œil. Nous sommes passés d'être un pays surtout générateur d'émigration à recevoir, au cours des deux dernières décennies, une quantité toujours croissante de citoyens étrangers qui choisissent le Chili comme l'endroit pour mener leur projet de vie. En comparant le phénomène actuel de l'immigration avec celui qui se produit dans d'autres pays - y compris certains de la région on peut conclure que le Chili présente un nombre faible d'immigrés, la population étrangère ne représentant qu'un petit pourcentage de la population totale du pays. (...) Jusqu'en 1982 le phénomène migratoire se caractérisa par la partie majoritaire de personnes d'origine européenne, l'existence d'un courant arabe et d'un autre, plus petit, d'Extrême orient. C'est seulement au cours des dernières décennies du $\mathrm{xx}^{\mathrm{e}}$ siècle que commence à prévaloir dans les courants migratoires vers le Chili la population des pays frontaliers à laquelle s'ajoute celle de pays asiatiques, qui sont le fruit, surtout, de la croissance économique que l'on commence à observer vers le milieu des années 1980. Après, avec l'avènement de la démocratie et la stabilité reconnue du Chili dans la région, cette tendance migratoire se consolide $»^{4}$. Après avoir donné certains chiffres (en 2002, 17\% des étrangers au Chili étaient de nationalités européennes, contre $67,8 \%$ de Sud-américains, l'estimation de la population étrangère selon le Ministère de l'Intérieur s'élève à 258000 personnes, les Péruviens représentent $26 \%$ de ce chiffre, les Argentins $22 \%$, ou encore la prépondérance des femmes chez les étrangers: $52 \%$ du total, mais plus de $60 \%$ chez les Péruviens, ou la distribution régionale: $63 \%$ dans la Région Métropolitaine, 7,8\% dans la $\mathrm{V}^{\mathrm{e}}$ Région de Valparaiso, 7,1\% dans la Ire Région de Tarapacá), il constate que: «Le Chili fait face aujourd'hui à une nouvelle immigration, très récente et en provenance particulièrement d'Amérique du Sud (d'origine andine). Ses principales caractéristiques sont sa spontanéité, sa finalité de travail essentiellement, avec un type de migrant dont la principale caractéristique est que sa provenance, ses coutumes sociales et de travail sont de type urbain, composée principalement de femmes et qui s'incorpore au pays en développant des activités comme main-d'œuvre, dans le domaine du bâtiment, de l'industrie et des services domestiques». Il arrive ensuite à la synthèse de la gestion migratoire au 
Chili par les quatre administrations de la Concertation: "Le gouvernement du Président Aylwin a réalisé le premier processus d'actualisation de la législation migratoire, en y introduisant d'importantes modifications, qui ont permis d'intégrer des dispositions d'accords internationaux assumés par le Chili, en rapport avec les réfugiés, et de créer un cadre de régulation qui réponde de façon cohérente à la plus grande mobilité que l'on a commencé à noter aux frontières et à l'intérêt croissant de citoyens étrangers pour connaître la réalité nationale. (...) Le gouvernement du Président Frei, face au défi de ce que l'on appelle aujourd'hui la "nouvelle immigration» a décidé de réaliser le premier et seul processus extraordinaire de régularisation migratoire, qui dans sa première étape a bénéficié à 44000 citoyens étrangers environ, avec des permis de séjour temporaires, et qui se consolida avec la remise d'environ 18000 permis de séjour définitifs. (...) Le gouvernement du Président Lagos a consolidé la modernisation de la gestion, aussi bien du point de vue politique que technique. Dans le premier il a créé une commission de politique migratoire qui a produit un texte qui résume la vision du gouvernement sur le phénomène des migrations. Ce document a été soumis à l'analyse et à la discussion de divers acteurs de la société civile, qui l'ont enrichi. (...) Les principes qu'il pose ont servi de base pour l'action de l'État pour ce qui concerne le phénomène migratoire. De là proviennent différentes actions et initiatives pour la régularisation de situations de séjour de citoyens étrangers, telles que:

- l'intégration des femmes migrantes enceintes au système de santé, à travers la régularisation de leur situation migratoire;

- la régularisation de la situation de séjour de tout enfant qui est inscrit dans un établissement scolaire reconnu par l'État;

- le respect du droit du travail pour les migrants: des campagnes de promotion menées ensemble par la Direction du Travail et le Département des Migrations pour faire respecter les droits en matière de travail des travailleurs étrangers au Chili;

- une initiative pour faciliter l'obtention du séjour de citoyens argentins au Chili, dans le cadre de l'Accord sur la résidence de nationaux de MERCOSUR, de la Bolivie et du Chili, et sur la Régularisation Migratoire sur le territoire des États membres et associés;

- une coordination entre le Service National des Mineurs et le Ministère de l'Intérieur afin de prévenir le trafic et la traite d'enfants étrangers au Chili; - dans le domaine du droit d'asile, on a souscrit un accord-cadre de coopération avec le Haut-Commissariat de Nations Unies pour les Réfugiés qui a permis de mener sept missions pour le transfert de réfugiés qui se trouvent dans plusieurs pays.

(...) En poursuivant le processus de modernisation de la gestion, la présidente Bachelet a mis dans son programme de gouvernement des références explicites à la thématique migratoire. (...) Cet engagement a au moins quatre composantes:

- une reconnaissance des soutiens que des milliers de Chiliens ont reçu à l'étranger, aussi bien à l'heure actuelle que dans une période antérieure. Dans l'optique de l'immigration, ceci est un engagement pour le Chili, qui devra regarder ce phénomène et proposer des politiques gouvernementales avec une vision de droits citoyens;

- l'impulsion d'une nouvelle législation pour les étrangers, qui intègre les engagements internationaux souscrits par le Chili;

- prendre en compte la variable migratoire dans les processus d'intégration régionale auxquels le Chili prend ou prendra part;

- le besoin d'intégrer la thématique de l'immigration dans le cursus scolaire $»^{5}$.

Bien entendu, les documents officiels ne donnent de la réalité qu'une image partielle et quelque peu idéalisée. Cependant, ils reflètent (et celuici ne fait pas exception à cette règle) un certain nombre d'orientations et traduisent un "air du temps» qui n'est pas sans rapport avec la situation concrète. Incontestablement, celui-ci s'inscrit dans la logique que Domenech appelle une "rhétorique de l'inclusion" et reflète une orientation globale de prise en compte de l'immigration, dans une optique d'intégration des populations concernées et de lutte contre les abus que celles-ci peuvent subir.

Au bout de ce rapide coup d'œil sur l'état de la question de l'immigration au Chili, quelques remarques simplement, en guise de conclusion:

a) Pour ceux qui s'intéressent un tant soit peu aux questions migratoires en France, je pense que la proximité et la distance entre les situations auxquelles sont confrontés les deux pays (et les immigrés dans les deux pays) ont pu être relevées au fil de cet exposé. Le Chili se trouve dans la situation d'être à la fois familier avec l'immigration et confronté à des situations inédites en la matière. Tant que la croissance et un bas taux de chômage seront au rendez-vous, on peut supposer que les difficultés ne seront pas insurmontables pour l'insertion sociale et professionnelle des nouveaux arrivants, mais il faut promouvoir (ou mieux matérialiser) des politiques d'intégration et un projet éducatif ayant pour objectif le respect de la diversité, la valorisation du vivre ensemble et la lutte contre les discriminations.

b) Dans un pays qui est transformé par une forte croissance économique et par un développement global incontestable (amélioration des conditions de vie, niveau éducatif et culturel plus élevé, infrastructures modernes, etc.) et où persistent de fortes inégalités sociales et économiques et des préjugés qui les alimentent et les légitiment, les réponses que l'on pourra apporter aux défis posés par l'immigration rejailliront sur des domaines autres que celui de l'immigration. On peut, à ce titre, prendre le cas de la situation des populations amérindiennes du pays, et d'abord celle des Mapuches, ou bien la question de l'éducation, qui prend depuis quelques années une place centrale dans le débat politique et plus largement dans le débat public, pour illustrer cette affirmation.

c) Enfin, la question migratoire se situe aussi au carrefour de plusieurs orientations sociétales qui débordent largement le cadre de cette question. 
Ainsi, les politiques sociales sont depuis plusieurs années un domaine qui se transforme, par la poussée exercée par les transformations des réalités matérielles et des mentalités et par la volonté, en tout cas pendant les gouvernements de la Concertation, de faire de ces politiques sociales un levier pour permettre l'amélioration des conditions de vie des populations les plus pauvres. Les situations spécifiques des immigrés ont obligé le pouvoir politique à se pencher sur des aspects de la couverture sociale qui étaient quelque peu délaissés, ou encore à se donner les moyens de corriger des orientations héritées des politiques autoritaires de la dictature. Dans ces deux cas, cela bénéficie aussi, en fin de compte, aux populations défavorisées du pays lui-même.

\section{Caractéristiques de l'immigration péruvienne}

Comme nous avons déjà pu le constater, l'immigration latino-américaine a largement pris la première place au Chili, depuis au moins une vingtaine d'années. Elle répond, comme les migrations antérieures et comme partout dans le monde, à des besoins objectifs de la société d'accueil (ici, le Chili) et des populations qui s'installent, provisoire ou durablement, dans le pays. Les avantages pour les uns et les autres (pays d'accueil et populations immigrées) dépassent largement les inconvénients, mais il faut rester lucides et attentifs vis-à-vis de ces derniers, notamment pour pouvoir mieux les maîtriser ou les surmonter. Examinons donc ces difficultés.

Il y a, d'abord, le fait que cette immigration en provenance de pays $\mathrm{du}$ sous-continent est relativement nouvelle (au moins par l'importance quantitative qu'elle atteint). Certes, avons-nous vu, l'immigration est assez largement constitutive du Chili actuel, mais d'une part elle avait beaucoup diminué dans la seconde moitié du vingtième siècle et, d'autre part, elle ne provenait pas des mêmes aires géographiques. Cela me semble suffire pour expliquer les raisons qui donnent à ce phénomène un caractère nouveau et les résistances qu'elle peut rencontrer. On peut, pour ce qui concerne les Péruviens et les Boliviens, faire appel à l'histoire des conflits qui ont émaillé les relations des trois pays (et surtout la guerre dite du Pacifique -1879/1883qui opposa le Chili au Pérou et à la Bolivie et qui a laissé des traces durables qui ressurgissent périodiquement, par la revendication, surtout bolivienne, de restitution des régions annexées par le Chili, ou, à l'heure actuelle, par le recours péruvien au Tribunal de La Haye pour modifier les limites maritimes fixées par des traités signés par les deux pays), mais aussi au caractère raciste de certaines images véhiculées concernant cette population de cholos (on peut traduire ce terme dans une première approximation par Métis, mais il est en fait «à géométrie variable») dont on dénonce la contribution, supposée ou réelle, à l'insécurité et à la délinquance. Certes, il ne faut pas généraliser et de telles réactions sont le fait d'une minorité de la population, mais les dérives sont toujours possibles, surtout si une crise économique devait survenir. À l'occasion du terrible tremblement de terre et du tsunami qui ont frappé le Chili en février 2010, on a relevé la situation difficile de nombreux Péruviens qui ont perdu leurs logements à Santiago ; on a alors parlé dans certains journaux des «150 000 Péruviens qui habitent la capitale». On peut également rappeler qu'un certain sentiment anti-chilien est présent au Pérou et en Bolivie, alimenté par les dirigeants politiques des deux pays, pour voir qu'il s'agit là d'une question complexe.

Il y a, ensuite, le statut d' ' indocumentado» (sans papiers) que partage une part importante de ces immigrés. En effet, les conditions d'obtention du titre de séjour pour un étranger n'est pas chose aisée, en dehors des cas plus classiques de conjoints de Chilien(ne) s, d'étudiants ou de salariés de firmes étrangères. À ce titre, on peut comparer les 195320 étrangers recensés en 2002 avec les 352344 personnes recensées en 2012, et avec les estimations donnant une fourchette entre 500000 et 800000 étrangers résidant au Chili qui sont le plus souvent considérées comme réalistes. Néanmoins, même les chiffres officiels reflètent cette tendance à l'augmentation rapide: du recensement de 1992 à celui de 2002 , le nombre d'étrangers vivant au Chili s'est accru de $75 \%$, et de 2002 à 2012 de $80 \%$... En même temps, on a observé une féminisation de l'immigration, surtout de la péruvienne: $63 \%$ des Péruviens étaient, en 2002, des femmes (pour les chiffres officiels, en tout cas), et d'autres nationalités sudaméricaines sont également à majorité féminine au Chili. Par ailleurs, comme nous le rappelle Paula Castillo Torres, «Le Décret-Loi 1094 (de 1975) règle l'entrée, la résidence, le séjour définitif, la sortie, le retour, l'expulsion et le contrôle des étrangers au Chili. Cependant celui-ci ne reconnaît pas la réalité des immigrés économiques, qui constituent une part importante des immigrés qui arrivent au Chili à l'heure actuelle, particulièrement pour ce qui concerne les personnes d'origine péruvienne, bolivienne et équatorienne. Plus, même: le Décret-Loi en question interdit l'entrée au pays aux personnes qui n'ont pas ou ne peuvent pas exercer leur profession ou métier, ou qui n'ont pas de ressources qui leur permettent de vivre au Chili sans constituer une charge pour la société. Ainsi, la normative chilienne ne reconnaît comme «résidents» que les individus qui ont un contrat, des étudiants, des travailleurs temporaires ou ayant l'asile politique ou le statut de réfugiés. (...) L'immigration au Chili se caractérise de nos jours par le fait qu'elle est fortement influencée par la variable travail. Les conditions auxquelles se voient confrontées ces personnes à leur arrivée au Chili, néanmoins, sont souvent attentatoires envers leurs droits humains. Ainsi, s'agissant du travail, on a noté que l'ouverture des frontières à la libre circulation du capital et des marchandises ne s'est pas accompagnée de la libre circulation des travailleurs. En fait, on constate que les plus grands abus auxquels se trouvent confrontés les immigrés sont dans le domaine du travail. Dans le cas spécifique des immigrés péruviens il est intéressant de noter que, bien qu'ils possèdent un niveau d'éducation assez élevé, les conditions de travail 
auxquelles ils font face restent toujours très précaires. (...) Les migrants péruviens travaillent dans les secteurs de moindre qualification, de plus basses rémunérations et de plus forts niveaux de risques ${ }^{6}$. Le constat de Castillo Torres est largement partagé, et des initiatives gouvernementales ont montré, depuis une bonne quinzaine d'années, que les pouvoirs publics ont pris conscience de cette situation et voulaient la modifier.

Du point de vue des immigrés, la situation est souvent vécue douloureusement. Nombre d'entre eux perçoivent une discrimination à leur égard, des attitudes de rejet. À ce titrelà, on peut rapporter les propos de María Emilia Tijoux, auteure d'une étude sur les immigré(e)s péruvien(ne) $s$ à Santiago: "Il est vrai que [le Chili] connaît une croissance en termes macroéconomiques, mais en même temps les inégalités économiques et sociales ont été rendues plus visibles. À cause de cela, dans un pays peu habitué à recevoir une grande masse de migrants sans ressources, il n'est pas rare que s'installe dans les secteurs même les plus pauvres de la société chilienne un sentiment de rejet et un processus de stigmatisation qui se développe et se caractérise par de multiples actes discriminatoires envers les Péruviens. Le chômage et l'exclusion ne sont pas caractéristiques d'un seul pays. Il s'agit de problèmes universels et pas tellement nouveaux, qui nous rappellent depuis la férocité de leurs conséquences, que les inégalités et les souffrances sociales continuent et que le racisme et la xénophobie font partie de ces conséquences-là ». Un peu plus loin, elle rapporte les propos de certains interviewés: "Je sais bien qu'ici aussi il y a des problèmes et je pense parfois que c'est pour ça que les gens nous méprisent et nous offensent, mais j'aimerais leur dire que nous sommes tous pauvres, que nous sommes tous des êtres humains, que nous avons un même Dieu et même une histoire semblable. Alors, qu'ils ne nous discriminent pas seulement à cause de notre couleur ou parce que nous sommes Péruviens. Nous ne leur avons rien fait de mal et on pourrait vivre bien mieux sans tant de discri- mination, tous ensemble» (Gladys, 54 ans, employée de maison). (...) «Au Chili nous faisons comme les autres, nous sommes employées de maison. (...) C'est vrai qu'ici on est un peu mieux économiquement, mais pas psychologiquement, le pays nous manque, la famille, le foyer. Ici tout change, la façon de vivre, même la façon de penser. (...) Des patrons, on peut dire qu'on a trouvé peu de gens conscients mais une grande majorité, je ne sais pas, c'est peut-être parce qu'on est Péruviens, ils nous traitent mal et il faut subir, on n'a pas où aller, on ne peut rien dire, alors on doit se mordre la langue et aller de l'avant. Je ne crois pas que nous prenons le travail de qui que ce soit, seulement il y a des gens qui n'aiment pas faire ce travail et nous, on le fait » (Sonia, 27 ans, technicienne en informatique et secrétaire comptable au Pérou, employée de maison, comme sa sœur qui est venue en même temps qu'elle) $»^{7}$. Ces témoignages, parmi d'autres, reflètent l'amertume ou le désarroi de certains immigrés péruviens; d'autres voient les choses sous un jour différent, surtout lorsque l'amélioration de leur sort est plus tangible. Certaines Péruviennes, arrivées au Chili avec un bagage scolaire relativement étoffé, commencent par travailler parfois comme employées de maison mais retrouvent par la suite une situation plus conforme à leur profil professionnel.

Les réseaux informels d'entraide sont efficaces mais ont une portée limitée; c'est pourquoi des associations se créent, encouragées par les pouvoirs publics, pour qui elles peuvent constituer un partenaire. Dès le début des années 2000, la volonté des pouvoirs publics s'est affirmée en la matière. Sous la première présidence de Michelle Bachelet (2006/2010), de nouvelles mesures ont été prises pour lutter contre les abus dont étaient victimes des immigrés (Péruviens et autres), pour renforcer l'efficacité du service social auprès de ces populations ou encore pour lutter contre la xénophobie. Certes, cela ne réussit pas à aplanir toutes les difficultés, car c'est aussi les mentalités qu'il faut faire évoluer, mais la position des pou- voirs publics est très importante en la matière.

Comme nous avons pu le constater dans le point précédent, l'immigration de ressortissants de pays limitrophes (et d'autres pays latino-américains, d'ailleurs) est, à la fois, un phénomène ancien et nouveau au Chili. Dans les villes du Nord, telles qu'Arica, Iquique ou Antofagasta, la présence de Péruviens est une donnée qui semble aller de soi depuis longtemps et ceux-ci développent des activités professionnelles diversifiées, allant d'emplois peu qualifiés (dans le secteur formel comme dans l'informel) à des occupations commerciales dynamiques et qui ont tendance à s'étendre. Ces Péruviens viennent de régions pas trop éloignées du Chili et, souvent, font des allers-retours fréquents entre leur domicile chilien et le péruvien. De la même façon, un nombre important de jeunes étudiants sont venus pendant longtemps de diverses universités péruviennes pour terminer leurs études au Chili ou pour faire une spécialisation ou un approfondissement, généralement dans les universités les plus prestigieuses de Santiago. Parmi eux, un certain nombre (une petite minorité, toutefois) a décidé de rester au Chili après la fin de leurs études, a épousé un (plus souvent, une) Chilien(ne) et a rejoint ainsi les rangs de la colonie péruvienne de la capitale.

Cependant, l'immigration péruvienne à laquelle nous allons nous intéresser n'est pas celle-là : c'est plutôt celle qui a fait son apparition vers la fin des années 1980 et qui s'accroît rapidement dès la seconde moitié des années 1990, qui a par ailleurs un profil bien différent de celui de sa devancière, du point de vue de l'origine géographique, socio-économique et culturelle, et qui met en œuvre des stratégies migratoires d'un type nouveau. À l'origine de cette évolution, il y a eu les crises (politique, sociale, économique) qui ont frappé le Pérou, notamment après la fin de l'expérience de réformisme militaire conduite par le général Juan Velasco Alvarado. L'extension des mouvements de guérilla (et d'abord celui du Sentier Lumineux, d'inspiration dite maoïste) a déstabilisé le pays et mis à nu les contradictions et 
les inégalités extrêmes, économiques, géographiques et ethniques tout à la fois. Le gouvernement Fujimori a démantelé les mouvements de guérilla et stabilisé l'économie, mais en tournant le dos aux promesses du candidat Fujimori et en menant un auto-coup d'Etat qui a ouvert la voie à de nombreuses exactions et à des scandales financiers. Depuis le départ contraint du dictateur, et sous les présidences d'Alejandro Toledo, d'Alan García et - à l'heure actuelle - d'Ollanta Humala, le Pérou a connu une amélioration réelle de sa situation mais les inégalités demeurent importantes.

Dans ce contexte, l'émigration est une sorte de soupape et elle est assez diversifiée en termes de pays d'accueil mais aussi de profils des émigrés. Le choix que font certains d'aller au Chili (mais aussi en Argentine, au Brésil ou, moins souvent, dans d'autres pays d'Amérique latine) est influencé à la fois par l'existence de réseaux informels qui font qu'on arrive dans un pays où on connaît des gens, mais aussi par la proximité géographique et une certaine familiarité avec la société d'accueil (langue, culture, modes de vie). Mais il ne faut pas perdre de vue l'hétérogénéité (en termes de classe sociale, de niveau d'études, d'âge, de sexe, d'état familial, etc.) de cette population, qui va donner lieu à des parcours migratoires différentiés. Cependant, jusqu'à un certain point, on peut parler à l'heure actuelle d'une "communauté péruvienne» au Chili, et notamment à Santiago. Comme nous le dit Carolina Stefoni, «(...) le fait qu'ils vivent dans des zones géographiques spécifiques et qu'il existe entre eux des relations humaines et sociales antérieures au processus migratoire, qui subsistent jusqu'à maintenant, nous permet de parler de la formation embryonnaire d'une «communauté». C'est peut-être ce fait qui a permis ou contribué à rendre plus visible l'immigrant péruvien. Il ne s'agit pas de personnes isolées, mais de gens qui appartiennent à un collectif, [qui ont] un sentiment de groupe, de communauté. Ceci pourrait engendrer dans la société réceptrice une certaine crainte au départ, car il ne s'agit pas d'individus atomisés mais d'un col- lectif qui se transforme en un "Autre» au sein de la culture dominante. (...) Mais la présence d'étrangers résidents engendre aussi une redécouverte de la spécificité chilienne, qui contraste avec la spécificité de ceux qui arrivent. La présence et l'identification de groupes humains provenant d'autres latitudes et avec des caractéristiques particulières (religion, langue, coutumes, etc.) fait naître des questions sur sa propre identité dans la mesure où l'identification d'un «autre» génère une redéfinition du «nous». Ce n'est pas seulement le cas de la société réceptrice, mais aussi de la communauté d'immigrants qui arrive et qui se consolide dans un lieu donné. Ces communautés aussi expérimentent des processus de redéfinition identitaire, dans la mesure où elles deviennent des communautés culturelles et sociales qui vivent et évoluent dans des pays et des cultures autres que les leurs. ${ }^{8}$. Il me semble, en effet, que le phénomène évoqué ici par Stefoni, la façon dont se joue la relation identité/altérité et les questions que pose celleci, sont bien un enjeu dans la société chilienne d'aujourd'hui. Récemment, deux faits divers ont nourri des commentaires et des réflexions qui illustrent les difficultés rencontrées par nombre d'immigrés latino-américains au Chili. Le premier concerne plutôt des Colombiens installés dans la ville d'Antofagasta (la plus importante du Nord du pays). Accusés d'alimenter la délinquance et l'insécurité dans cette ville, ils ont été la cible d'un collectif anti-colombien à l'accent ouvertement xénophobe et raciste, qui revendiquait plusieurs centaines de membres et qui a convoqué une manifestation en octobre 2013 pour dénoncer les «activités maffieuses» de cette population, qui serait responsable de l'essor du trafic de drogue, des crimes, des vols et de la prostitution, notamment. La manifestation a été interdite par l'intendente (équivalent du Préfet en France) mais une cinquantaine de personnes se sont réunies dans la principale place de la ville pour crier des consignes, faisant face à des contre-manifestants, qui étaient également une cinquantaine, dénonçant l'amalgame "colombiens = délinquants » à l'origine de cette initia- tive. Il faut dire que, selon des sources concordantes, les Colombiens résidant à Antofagasta sont entre huit et dix mille personnes (quinze mille dans la II Région, dont Antofagasta est la capitale), et que leur visibilité est forte, aussi bien par leur nombre (la ville a une population d'environ 380000 personnes) que par leurs caractéristiques ethniques et culturelles.

Le deuxième a été la mort (en avril 2013) d'un jeune Péruvien, poignardé à l'entrée d'une discothèque, fréquentée essentiellement par des Péruviens, dans un quartier populaire du nord de la ville. La famille de la victime a rendu responsable du crime des supporters d'un club péruvien (Universitario), qui se seraient pris à lui car il était un ardent supporter d'un club rival de Lima (Alianza). Même si cette thèse a été démentie par les auteurs, ce drame a mis en lumière l'existence de groupes de supporters de ces clubs qui mèneraient des activités à Santiago et s'affronteraient ouvertement dans les quartiers où réside un fort contingent de Péruviens. Des reportages ont été consacrés à cette question, notamment à la télévision, où des habitants (chiliens) de ces quartiers dénonçaient l'insécurité que ces bagarres y faisaient régner, et se plaignaient de l'immobilisme des autorités sur la question. En novembre dernier, un Péruvien (supporter d'Universitario) était assassiné dans le centre de la ville par des jeunes (Chiliens, cette fois-ci) qui revêtaient des maillots de Colo Colo. Il faut savoir que les deux grands clubs péruviens entretiennent des rapports amicaux avec Universidad de Chile (pour ceux d'Universitario) et Colo Colo (pour ceux d'Alianza), qui sont également les deux clubs les plus populaires du Chili.

Il ne faut, certes, pas exagérer la portée de ces faits divers, mais ils nous parlent à leur façon des dangers que présente une prise en compte insuffisante des questions soulevées par l'insertion (et l'intégration) des populations immigrées les plus vulnérables. Bien entendu, tous les Péruviens au Chili ne sont pas à ranger dans la catégorie des populations vulnérables, mais c'est le cas d'une fraction importante d'entre eux. Parce qu'ils (ou elles) n'ont pas de papiers, 
et/ou parce qu'ils exercent des métiers parmi les moins qualifiés, qu'ils ont un niveau scolaire faible, qu'ils occupent des emplois précaires, qu'ils habitent des logements exigus ou vétustes, dépourvus souvent du minimum de conditions d'hygiène et de confort, ces hommes et ces femmes ont bien des écueils à surmonter pour pouvoir être en conformité avec les attentes qui les ont amenés à émigrer. Certes, il n'y a là rien de bien nouveau par rapport à ce qu'ont connu (et connaissent) bien des migrants de par le monde, mais cela ne pose pas moins pour autant des questions à la société chilienne actuelle.

\section{Le travail domestique et le care: distinctions et aspects communs}

Les chaînes globales de soins, dont nous examinons ici une figure particulière, sont un phénomène en pleine expansion dont on évoque surtout les modalités de réalisation dans les pays récepteurs, et surtout dans les plus riches d'entre eux. Pendant longtemps, la migration transnationale des femmes n'a été perçue que comme une conséquence découlant de celle des hommes, et les immigrées restaient «invisibles» pour les différents acteurs qui s'intéressaient aux questions migratoires, ce qui était plutôt une conséquence de l'idéologie dominante qu'un reflet des situations objectives. En effet, quantitativement elles étaient une part importante des migrants (rarement moins de 35 ou de $40 \%$ ), et qualitativement leur rôle n'était pas moins crucial, à la fois parce que beaucoup d'entre elles travaillaient hors de leur foyer et parce que l'éducation, les soins et la socialisation des enfants étaient de leur responsabilité et donc une part importante du succès ou de l'échec de l'intégration reposait sur leurs épaules. $\mathrm{Si}$ on ne parlait pas encore de care, nombre de ces femmes travaillaient dans des foyers plus ou moins aisés du pays d'accueil, s'occupant de ménage, mais aussi de soins aux enfants, aux malades ou aux personnes âgées qui y vivaient. Cependant, tout semble indiquer qu'il y a un nombre croissant de femmes étrangères qui travaille dans ce secteur d'activité, celui-ci connaissant d'ailleurs une forte croissance. La migration de femmes seules, qu'elles aient laissé leur famille au pays ou qu'elles soient seules (ou en tout cas sans responsabilités familiales), est aujourd'hui plus forte que celle d'hommes seuls pour plusieurs nationalités, et d'ailleurs les Péruviennes, avons-nous vu, constituent au Chili plus de $60 \%$ du total des Péruviens y résidant.

Dans les nombreux travaux consacrés à la notion de care qui ont vu le jour, notamment, au cours des vingt dernières années, on retrouve souvent une analyse de la difficulté de rendre compte des contours qu'a celui-ci, dans la mesure où s'y retrouvent imbriqués le formel et l'informel, le matériel et l'affectif, des activités et des attitudes, la sphère domestique et des équipements extérieurs à celle-ci, l'univers familial et celui de diverses institutions, le travail «bénévole» et le travail rémunéré, pour ne citer que certaines des dichotomies (les termes de celles-ci pouvant être ou devenir concomitants ou antagonistes). Ce qui nous intéresse ici c'est, d'une part, la question des chaînes globales, et d'autre part celle des frontières et des chevauchements du travail domestique et du care, et à ce titre nous pouvons emprunter la proposition faite par Irma Arriagada et Marcela Moreno: «Par soins nous entendons la gestion et la création de ressources pour le maintien quotidien de la vie et de la santé des personnes; la fourniture quotidienne du bien-être physique et émotionnel, qui satisfait leurs besoins tout au long de tout cycle vital. Le soin se réfère aux biens, aux services et aux activités qui permettent aux personnes de s'alimenter, de s'éduquer, d'être en bonne santé et de vivre dans un habitat approprié. La distinction entre ce que l'on considère être de l'ordre du soin et du travail domestique est difficile à dessiner et entre les deux il y a des zones grises qui se superposent. (...) Le soin est toujours une activité qui implique une relation de face-àface entre deux personnes, alors que le travail domestique peut se réaliser sans cette relation. On considère que la catégorie travail de soins peut inclure le travail domestique et que tous les deux peuvent se faire de façon rémunérée ou non rémunérée ${ }^{9}$. Le terme care étant traduit en espagnol par cuidado, ceci explique que dans cette citation le mot care soit absent.

Au Chili comme en France - même si chaque société a ses propres caractéristiques et des modes singuliers d'expression de ce phénomène -, la situation et le statut social et juridique des femmes ont connu d'importantes transformations au cours du dernier demi-siècle. Pour ce qui concerne la société chilienne, l'exode rural et la croissance urbaine, les progrès en matière d'allongement et de diversification de la scolarité des filles, les changements démographiques (et notamment l'allongement de l'espérance de vie, la chute rapide de la mortalité infantile et la forte diminution de la fécondité), une augmentation des emplois dans le secteur tertiaire (administration, commerce et autres services), l'évolution aussi des mentalités et des modes de vie, ont amené un nombre croissant de femmes à rechercher une insertion permanente dans la vie professionnelle et ont créé de nouveaux besoins en termes de remplacement des activités domestiques qu'elles n'étaient plus en mesure d'assurer. Certes, dans les catégories plus aisées de la population, mais aussi dans un nombre important de foyers des classes moyennes, le recours à des employé(e)s de maison était fréquent et ces emplois étaient le plus souvent occupés par des femmes ayant émigré de la campagne, seules ou avec leur famille. Cependant, progressivement et au fur et à mesure que d'autres opportunités d'emploi se sont développées dans le secteur industriel et celui des services et qu'elles ont connu de nouvelles aspirations, l'emploi domestique leur est apparu comme un pis-aller ou comme un moyen provisoire de subvenir à leurs besoins, tout en cherchant une autre forme d'insertion professionnelle plus satisfaisante à leurs yeux, plus compatible aussi avec une vie de famille. Dès lors, alors que les besoins augmentaient, les candidates à ce type de poste se faisaient moins nombreuses, ce qui a eu pour 
effet bénéfique d'améliorer les conditions de travail et le niveau de rémunérations de celles qui occupaient ces postes. On peut ainsi expliquer comment s'est produite l'évolution qui a ouvert largement ce secteur d'activité à des immigrées (les Péruviennes étant les plus nombreuses parmi celles-ci dans ce segment du marché du travail). Mais il y a là également des dangers et des aspects négatifs qui apparaissent. On peut ici reprendre l'analyse que fait, pour le cas de la France, Sabah Chaib, en parlant de "l'emploi des femmes immigrées, laboratoire de la flexibilité de la main-d'œuvre», où il dit que «La tertiarisation de l'économie entraîne le marché du travail dans un double mouvement: élévation du niveau moyen de qualification et fragilisation plus grande liée à une intégration moins assurée pour les travailleurs qui se retrouvent au bas de l'échelle. Cependant, les prospectives montrent que les caractéristiques de l'activité féminine vont aller en se détériorant: un taux d'activité sans cesse croissant mais accompagné d'une forte ségrégation de l'emploi, une bipolarisation accrue entre femmes qualifiées et non qualifiées; une hétérogénéité croissante des femmes et de leurs parcours; à ce titre, on parle d'une individualisation très forte des parcours; ce n'est plus l'emploi qui porte l'individu mais l'individu qui "porte», construit son emploi. (...) Dans ce tableau sombre des emplois non qualifiés, la main-d'œuvre immigrée du fait de son positionnement a expérimenté en premier ces emplois et/ou les nouvelles formes d'emplois (externalisation, sous-traitance, vente à domicile, travail à domicile, horaires flexibles, contractualisation sous diverses formes). Les enquêtes qualitatives sur les femmes immigrées montrent des parcours marqués par la flexibilité des temps de travail et la précarisation des formes de contractualisation avec les employeurs. (...) À l'intérieur des emplois féminins, les emplois occupés par les femmes immigrées, au bas de l'échelle pour la majorité, préfigurent les évolutions du marché du travail en ce qui concerne les emplois non qualifiés. (...) Si les secteurs d'activité féminins sont repré- sentatifs des recompositions du marché du travail, les emplois occupés par les femmes immigrées le sont encore plus du point de vue des formes d'emplois (...) Ainsi, à regarder de près les tendances d'activités des femmes immigrées, ces dernières semblent s'inscrire dans le même processus que les hommes immigrés (amortisseur de crise/flexibilité du travail, révélation d'emplois ou de formes nouvelles d'emploi), à la différence près que les secteurs d'activité où elles se concentrent aujourd'hui comme hier, sont moins visibles que les hommes immigrés parce qu'éclatés dans un secteur d'activité très hétérogène ${ }^{10}$. Voilà bien un danger qui menace aussi la société chilienne: les femmes immigrées connaissent, en quelque sorte, un avant-goût de ce que pourrait être l'évolution du monde du travail pour les secteurs les moins bien pourvus de la société. Certes, l'analyse de Chaib s'applique à une société en panne de croissance, ce qui n'est pas le cas du Chili, mais les filets de protection pour les populations plus vulnérables y sont bien plus faibles et la tendance à cette dualisation correspond bien au modèle néolibéral qui marque encore de nos jours les orientations économiques de ce pays.

L'intérêt croissant que suscite le care dans le débat politique et le champ universitaire n'est pas le fruit du hasard ou d'une mode quelconque. Pour Sonia Montaño, «Ceci est sans doute dû à des facteurs démographiques tels que la longévité et la qualité de vie des hommes et des femmes, la transition démographique qui fait que les femmes passent de prendre soin des enfants à prendre soin des personnes âgées, les transformations familiales, les chaînes globales de care dans lesquelles la migration de femmes acquiert de l'importance pour l'envoi de fonds et la subsistance familiale et, enfin, mais ce n'est pas le moins important, parce que les femmes plus ou moins éduquées veulent leur autonomie économique, avoir des revenus propres et mettre à profit le capital éducatif obtenu. Ce que l'on appelle la crise du care n'est rien d'autre qu'un symptôme de l'émancipation des femmes ${ }^{11}$. Les premiers entretiens que j'ai pu réaliser à Santiago ${ }^{12}$ confirment l'importance que revêt pour les travailleuses familiales péruviennes le fait de pouvoir envoyer de l'argent à la famille restée au pays (souvent le conjoint et les enfants, mais aussi les parents et d'autres membres de leur famille), même si dans un certain nombre de cas elles ont leur famille la plus proche qui les a rejoint ou qu'elles ont rejoint au Chili. Mais même dans ce cas, souvent elles envoient de l'argent à ceux qui sont restés (en général, leurs parents) et/ ou mettent de côté de l'argent pour un projet qui vise le retour. Quant à la question de la recherche d'une autonomie matérielle, elle concerne à la fois les pourvoyeuses de soin et leurs employeures. Pour ces dernières, le recours à une travailleuse familiale est (pour celles, en nombre croissant, qui exercent une activité professionnelle) une condition nécessaire pour pouvoir poursuivre une activité professionnelle hors de leur foyer. Vu sous cet angle, on pourrait dire que le care, sous la forme que nous l'examinons ici, fait l'affaire de toutes les personnes concernées par la relation que celui-ci engendre. Mais c'est là une vision pour le moins tronquée de la question, car le travail domestique salarié est une forme de rapport où l'exploitation et l'asservissement des unes (les salariées) ne saurait être plus acceptable parce que ce travail permet l'affranchissement et l'épanouissement des autres (les patronnes); c'est seulement si l'on veille strictement à la mise en place, puis au respect, d'une réglementation veillant à défendre les droits et la dignité de ces travailleuses, à éviter aussi les abus de toute sorte dont beaucoup d'entre elles sont victimes, que l'on pourra défendre le caractère nécessaire et globalement positif du care (du moins sous la forme où nous l'examinons ici).

$$
\begin{aligned}
& \text { Les chances } \\
& \text { et les dangers que }
\end{aligned}
$$




\section{comporte l'extension du travail du care}

Dans ce dernier point, je vais aborder ce qui me semble relever des aspects positifs et négatifs, potentiels ou réels, de cette évolution, en rappelant d'abord que le soin a toujours au moins trois acteurs concernés: les soignants, les soignés et la société globale (censée être tant bien que mal représentée par les pouvoirs publics). D'autres acteurs sont souvent tout aussi impliqués dans la relation: les familles et le monde associatif, principalement. Les intérêts et points de vue de ces différents acteurs ne sont ni naturellement convergents ni foncièrement opposés, mais il est bon de se rappeler qu'ils sont distincts. Les soignants (ici, les travailleuses familiales péruviennes dans la mesure où elles sont concernées par le care) feront l'objet de mon attention plus particulière. J'emploierai le mot au féminin, étant entendu qu'il s'agit de femmes dans l'écrasante majorité des cas.

Les soignantes, donc, trouvent par l'accroissement des besoins en matière de care des opportunités d'emploi et une certaine forme de reconnaissance par la dimension affective et relationnelle que prennent les tâches qu'elles accomplissent au sein des foyers qui ont recours à elles. Cependant, le care exercé par des employées de maison est intégré à l'ensemble des tâches domestiques, dans un rapport hiérarchique d'une patronne et de sa bonne, où cette dernière va se trouver d'autant plus désavantagée qu'elle est étrangère, peu au courant des droits qui sont les siens, souvent même en situation de séjour irrégulière. Certes, les réseaux informels que la plupart d'entre elles tissent et les associations qui les accompagnent jouent un rôle pour réduire cette vulnérabilité, mais la relation avec l'employeur(e) reste dissymétrique. Lorsque les tâches soignantes prennent une part significative dans la charge de travail des travailleuses familiales, c'est leur reconnaissance qui est en jeu, aussi bien en termes d'émoluments que de droits (aux congés, à la formation, etc.). Il faut veiller, en somme, à ce que l'on prenne aussi soin des soignantes!

Il ne faut pas oublier que, pour ces femmes venues de loin notamment, leur disponibilité pour s'occuper de la maison, des enfants, des malades, des personnes âgées, dans cette famille qui n'est pas la leur, a pour pendant (lorsqu'elles sont elles-mêmes mères de famille, et c'est ainsi dans la majorité des cas) qu'elles doivent renoncer à s'occuper de leur foyer, de leurs enfants, des autres membres de leur famille aussi, et qu'il leur faut trouver les arrangements qui vont leur permettre de quitter leur foyer de façon provisoire (mais il s'agit parfois d'un provisoire qui dure longtemps). Ces chaînes globales $d u$ care ne font qu'entériner et actualiser de vieilles inégalités: les pauvres sont au service des riches, les femmes sont au service des hommes et «la raison du plus fort est toujours la meilleure».

Par ailleurs, la question se pose également d'un renforcement de la protection des droits des travailleuses familiales en général, qu'elles soient étrangères ou Chiliennes, qu'elles aient ou non une part de leur travail consacrée au care. Il s'agit d'abord de faire appliquer la législation existante en matière de salaires, de cotisations sociales, d'horaires de travail, de paiement des heures supplémentaires, de droit au repos, de recours contre toute forme d'abus ou d'arbitraire. Il s'agit ensuite de compléter la législation existante pour qu'enfin elles soient considérées comme des travailleuses comme les autres, et non plus comme les héritières des servantes qui, encore dans un passé récent, étaient des femmes taillables et corvéables à merci. Au mois de novembre dernier, des manifestations de travailleuses familiales (appelées «asesoras del hogar », littéralement conseillères (ou assistantes) du foyer, mais nommées familièrement «nanas», que l'on peut traduire par nounous) ont eu lieu au Chili, à l'appel d'un syndicat et d'associations, pour des revendications qui relèvent des deux dimensions ici nommées. À Santiago, les manifestations se sont déroulées dans les «beaux quartiers» de la capitale, pour se rappeler au bon souvenir de leurs employeur(e)s...

Du point de vue des employeur(e) $s$, et notamment des femmes salariées appartenant aux classes moyennes, le recours à des travailleuses familiales est souvent un choix contraint, dans la mesure où des structures collectives d'accueil pour leurs jeunes enfants n'existent pas ou sont trop chères ou de trop mauvaise qualité, et qu'il en va de même pour les personnes âgées dépendantes ou les handicapés. Il s'agit là d'une sorte de cercle vicieux, puisque l'absence de structures adéquates alimente le travail domestique rémunéré, qui à son tour sera mis en avant pour que celles-ci ne soient pas considérées nécessaires... On n'en sortira, vraisemblablement, que par la revalorisation des salaires de ces employées et par l'application de mesures de contrôle concernant leurs conditions de travail; dans le discours des pouvoirs publics, la volonté d'avancer sur ce dossier existe, mais il reste à vérifier si les actes seront au diapason de ces paroles. Le contexte sociodémographique et économique est favorable pour que des avancées se produisent en la matière, mais des résistances de diverse nature sont à surmonter.

On peut se poser également la question des effets que ces migrations de femmes mères de famille entraînent dans leur groupe familial et dans la société d'origine, même si elle n'apparaît souvent qu'en filigrane dans les entretiens que j'ai eus, comme si en parler pouvait remettre en question la légitimité de leur décision de partir. Il serait, par exemple, intéressant de voir dans quelle mesure les stéréotypes sexuels et les rapports de pouvoir entre conjoints se trouvent (ou pas) modifiés par ces migrations de femmes (qui, parfois, à l'image de ce qui était le cas pour les hommes qui émigraient en laissant leur famille au pays, font venir plus tard leur mari et/ ou leurs enfants). Par ailleurs, ces effets se feront sentir par l'importance que prend, pour un nombre important de femmes migrantes, la maternité à distance et ce que cela peut entraîner au niveau de leurs enfants. C'est là aussi vouloir sortir de l'ethnocentrisme 
de sociétés nanties qui ne semblent concernées que par leurs soucis et leur confort. Marcela Cerrutti et Alicia Maguid, dans une recherche qu'elles ont menée concernant la migration sud-américaine vers l'Espagne, nous disent que "Le regard de genre est indispensable pour comprendre non seulement pourquoi se produit ce transfert global des soins et pourquoi se sont formées ces familles divisées ou transnationales, mais aussi [...] pour éclairer la dynamique organisatrice de la globalisation. (...) Il faut faire porter l'analyse sur ces circuits de femmes qui traversent des frontières, pour comprendre les dynamiques de genre présentes dans l'économie globale; ce qu'on appelle «la féminisation de la survie» fait allusion aussi bien à la dépendance croissante du travail des femmes pour les familles et les communautés d'origine et de destination, comme à celle des États, qui délèguent en elles les fonctions de soins ${ }^{13}$. On voit bien ici l'intérêt qu'il y a à croiser l'approche monographique et celle qui s'intéresse aux grandes tendances et dimensions de cette question, qui concerne les disciplines qui s'intéressent aux migrations, à la famille, aux relations de genre, au travail, aux politiques publiques ou aux relations interculturelles.

L'immigration, comme reflet de l'écart croissant entre sociétés riches et sociétés pauvres, est porteuse de tensions et de conflits, dès lors qu'elle est utilisée pour freiner les mutations sociales, économiques et culturelles nécessaires, aussi bien dans les pays d'origine de ces migrants que dans ceux qui les accueillent. L'Espagne est, à ce titre, un cas intéressant à étudier, car l'arrivée massive d'immigrés (d'Amérique du Sud, mais aussi d'Afrique et d'Europe de l'Est) a fourni une main-d'œuvre dans différents secteurs où il y avait pénurie, tels que l'emploi domestique, mais aussi l'agriculture, le bâtiment ou le tourisme, avant que le pays ne s'enfonce dans une grave récession, avec des niveaux de chômage très élevés. Dans ce contexte, les immigrés sont, bien sûr, les premiers touchés; le secteur du care et des emplois domestiques n'est pas le plus mal loti, mais il n'est pas non plus préservé des effets de la crise globale qui touche l'Espagne, et pour ces femmes venues de loin c'est bien souvent très problématique, surtout lorsqu'elles sont privées de ressources, que leurs enfants sont restés au pays et qu'elles ne peuvent pas continuer à leur envoyer de l'argent.

La réflexion autour des objectifs et des moyens pour mettre en place une politique globale du care est aujourd'hui nécessaire, et pas seulement au Chili. Comme l'écrivent Arriagada et Moreno, «L'externalisation des soins familiaux, [transférant ceuxci] de la famille aux services publics ou au marché du travail, comme stratégie adaptative à l'augmentation de la demande de soins, n'a pas changé la configuration traditionnelle de la division sexuelle du travail, car ce sont toujours des femmes qui effectuent le travail domestique et de soins, tant dans la famille que sur le marché. ${ }^{14}$. On pourrait également ajouter qu'à la reproduction de la division sexuelle du travail s'ajoute une reproduction sociale et ethnique et qu'il y a aussi, dans le choix du recours au marché plutôt qu'à des institutions publiques ou associatives, une influence claire des options idéologiques néolibérales dont la conséquence est le renforcement des inégalités. C'est pourquoi on peut souhaiter qu'à l'heure où, au Chili, la réduction des inégalités sociales et économiques prend une place centrale dans le débat politique, on élargisse ce débat au domaine du care. Bien entendu, les problèmes qui se posent ne sont pas les mêmes quand on passe des soins aux jeunes enfants aux soins aux malades, à la prise en charge des handicapés ou des personnes âgées dépendantes; il faut examiner les situations concrètes, trouver les réponses les mieux adaptées pour chaque catégorie de population concernée, voir aussi si les actions souhaitables sont compatibles avec les moyens existants. Il ne faut pas non plus avoir l'illusion qu'un changement profond peut se faire en un court laps de temps. Mais l'absence de débat ou les discussions techniques qui occultent la portée des choix à faire ne font pas avancer ni la réflexion ni l'action en la matière. Surtout, ici comme dans d'autres domaines, il faut que la discussion qui précède l'élaboration des politiques et des dispositifs associe les acteurs de terrain, associations, professionnels et syndicats.

Si on s'engage dans cette voie, et si des progrès significatifs y voient le jour, on pourra dire - d'une certaine façon - que le care contribue, comme l'appelaient de leurs voux les précurseur(e)s des travaux théoriques et empiriques de la "société du care», à l'émergence de sociétés plus justes et fraternelles.

\section{Notes}

1. Nous employons les termes concertationnisme ou concertannioniste comme traduction littérale des mots utilisés pour désigner les politiques et actions conduites par la Concertation des Partis pour la Démocratie.

2. Domenech (E.), «La "nouvelle politique migratoire" en Argentine: les paradoxes du programme "Patria Grande" ", Problèmes d'Amérique latine, $\mathrm{n}^{\circ} 75$, Hiver 2009/2010, Choiseul éd., p. 37-59.

3. Ces chiffres sont extraits d'un document officiel de la DEM, Evolución de la gestión Gubernamental desde 1990: Desarrollo del fenómeno de las Migraciones en Chile, 2008, DEM, Ministerio del Interior, in http://www.extranjeria.gov.cl/filesapp/ migraciones.pdf

4. Ibid.

5. Ibid.

6. Castillo Torres P., Movimientos de migración, emigración e inmigración, in monografías.com

7. Tijoux M.E., «Morderse la lengua y salir adelante. La dificultad de ser peruano en Chile», Amérique latine. Histoire et Mémoire. Les Cahiers ALHIM, n 5, 2002.

8. Stefoni E. C., Inmigración peruana en Chile. Una oportunidad a la integración, 2003, Santiago, Ed. Universitaria, p. 26-27.

9. Arriagada I. et Moreno M., «La constitución de cadenas globales de cuidado y las condiciones laborales de las trabajadoras peruanas en Chile», in Stefoni C., ed., Mujeres inmigrantes en Chile. ¿Mano de obra o trabajadoras con derechos?, 2011, Santiago, Ediciones Universidad Alberto Hurtado, p. 151-191.

10. Chaib S., «Femmes, migrations et marché du travail en France», Les Cahiers $d u$ CEDREF, $\mathrm{n}^{\circ} 12,2004$, p. 211-237.

11. Montaño S., "El cuidado en acción», in Montaño S. et Calderón C., coord., El cuidado en acción. Entre el derecho y el tra- 
bajo, Cuadernos de la CEPAL, $\mathrm{n}^{\circ}$ 94, 2010, Santiago, Naciones Unidas éd., p. 26.

12. Dans le cadre de la recherche que je mène sur les femmes immigrées péruviennes au Chili, j'ai pris contact avec plusieurs structures associatives qui viennent en aide à ces migrantes et, par leur truchement ou par des contacts informels, j'ai rencontré quatre de ces femmes, dont trois mères de famille et une célibataire, qui travaillent toutes comme employées de maison, deux des mères en question ayant leurs conjoints et leurs enfants au Pérou. Cette recherche étant encore dans une phase peu avancée, je n'ai pas utilisé pour cet article les entretiens en question, cela fera l'objet d'une publication ultérieure.

13. Cerrutti M. et Maguid A., Familias divididas y cadenas globales de cuidado: la migración sudamericana a España, Serie Políticas Sociales, $n^{\circ} 163,2010$, Santiago, Ed. CEPAL, p. 14.

14. Arriagada I. et Moreno M., op. cit., p. 159. 
